\title{
Anchoring PEG-oleate to cell membranes stimulates reactive oxygen species production
}

\author{
Shinji Sakai*, Koujiro Nomura, Kei Mochizuki, Masahito Taya
}

Division of Chemical Engineering, Department of Materials Engineering Science, Graduate School of Engineering Science, Osaka University

1-3 Machikaneyama-cho, Toyonaka, Osaka 560-8531, Japan

*Corresponding author: Shinji Sakai

E-mail: sakai@cheng.es.osaka-u.ac.jp

Tel.: +81-6-6850-6252, Fax: +81-6-6850-6254

Statistical summary -Total number of words: 4709; Number of Figures: 7, Number of Table: 1. 


\section{ABSTRACT}

Polyethylene glycol (PEG) derivatives possessing oleyl and reactive groups for conjugating functional substrates, such as proteins and quantum dots, are useful materials for cell-surface engineering and cell immobilization onto substrates. The reagent is known as a biocompatible anchor for cell membranes (BAM). Here, BAM-anchoring on cell membranes is reported to stimulate reactive oxygen species (ROS) production in those cells. Significant increases in ROS production and release to the surrounding environment were detected in mouse fibroblast cell line 10T1/2 when soaked in a solution containing BAM conjugated with $1 / 10 \mathrm{~mol} / \mathrm{mol}$ bovine serum albumin at $1.5 \mu \mathrm{M}$-protein. ROS production stimulation was confirmed to be independent of the protein crosslinked with BAM and of cell type. Similar stimulation was detected for BAMs conjugated with ovalbumin and casein, human hepatoma cell line HepG2, and human umbilical vein endothelial cells. Considering the effects of ROS on a variety of cellular processes, these results demonstrated the necessity for focusing attention on the effects of generated and released ROS on the behaviors of cells in the studies applying BAM to cells.

Keywords: Reactive oxygen species; Hydrogen peroxide; Biocompatible anchor for cell membrane;

Oleic acid; PEG-oleate; Cell surface modification

\section{Introduction}

Polyethylene glycol (PEG) derivatives possessing oleyl and reactive groups have been used as useful reagents for cell surface modification [1-5] and immobilization of cells onto a variety of material surfaces [6-9]. The oleyl group acts as a hydrophobic cell-membrane anchor. Varieties of molecules and 
materials, including fluorescent proteins [4], enzymes [5], and quantum dots [10], have been conjugated with PEG derivatives, via cross-linking with a reactive group, for immobilizing them onto cell surfaces (Fig. 1). The biocompatibility of PEG derivatives has been confirmed by measurements of cell viability and subsequent growth after modification of their surfaces [4-7]. Because of their reported superior biocompatibility, PEG derivatives are called "biocompatible anchors for cell membranes” (BAM).

In this study, the effect of applying BAM modifications to cells was studied in terms of the resulting stimulation of reactive oxygen species (ROS) production. A better understanding of subsequent events caused by BAM-anchoring could help develop a better interpretation of individual results in the studies applying BAM to cells. Considering BAM applications in a wide range of cell studies, this investigation into ROS generation was important because ROS have been implicated in regulation of a variety of cell behaviors, such as adhesion, migration, and proliferation $[11,12]$. Moreover, ROS have been associated with activation of metalloproteinases [13, 14], transcription factors [15, 16], and proto-oncogene expression [17]. However, the effects of BAM-anchoring on cells from the aspect of ROS production stimulation have not been previously investigated. The oleyl group in BAM that anchors in cell membranes was hypothesized here to stimulate ROS production. Oleic acid is a fatty acid that is the most widely distributed and abundant in nature, being found in both animal fats and vegetable oils. Oleic acid has been reported to produce a variety of beneficial effects, including effects on anti-aging [18], autoimmune and inflammatory diseases, [19], wound-healing enhancement [20], and protection from cancer [21]. At a cellular level, it has been reported that some fatty acids stimulate ROS generation via activation of NADPH oxidase present in membranes [22, 23]. Induction of ROS generation by oleic acid has been reported in cells, such as neutrophils [24], fibroblasts [22], vascular smooth muscle cells [25], and endothelial cells [23]. The regulation of the cellular redox state by fatty acid-induced stimulation of NADPH oxidase activity is considered to have important effects on redox-sensitive 
signaling cascades [21]. To investigate the effect of BAM-anchoring, changes in intracellular ROS output were measured using a fluorescent probe for detecting $\mathrm{H}_{2} \mathrm{O}_{2}$. In addition, ROS release to the surrounding environment was investigated by a method involving horseradish peroxidase (HRP)-catalyzed, hydrogel-sheath formation caused by consumption of released $\mathrm{H}_{2} \mathrm{O}_{2}$ [26]. It has been reported that $\mathrm{H}_{2} \mathrm{O}_{2}$ is the principle $\mathrm{ROS}$ induced by oleic acid [25]. The effects on cell viability and growth after BAM-anchoring were also investigated under conditions that enhanced ROS production.

\section{Materials and methods}

\subsection{Materials}

BAM possessing a terminal $N$-hydroxysuccinimide (NHS) activated group (BAM-NHS, PEG: MW8,000, Sunbright ${ }^{\circledR}$ OE-080CS) was purchased from NOF Corp. (Tokyo, Japan). Oleic acid, bovine serum albumin (BSA), casein, ovalbumin, HRP, and the cell membrane permeable fluorogenic probe 3'-O-Acetyl-6'-O-pentafluorobenzenesulfonyl-2'-7'-dichlorofluorescien $\left(\mathrm{BES}-\mathrm{H}_{2} \mathrm{O}_{2}-\mathrm{Ac}\right)$ were purchased from Wako Pure Chemical Industries, Ltd. (Osaka, Japan). Green fluorescent protein (GFP) was obtained from GFP-expressing Escherichia coli, as previously reported [27]. Alginate derivative possessing both phenolic hydroxyl $(\mathrm{Ph})$ moieties and fluorescein moieties (Flu-Alg-Ph) was prepared by conjugating sodium alginate (MW 70,000, Kimica I-1G, KIMICA, Tokyo, Japan) with tyramine (Tokyo Chemical Industry Co., Ltd., Tokyo, Japan) and 4-aminofluorescein (Sigma-Aldrich, Inc., St. Louis, MO, USA) through aqueous-phase carbodiimide activation chemistry, based on a previously reported method [28]. Mouse fibroblast cell line 10T1/2 and human hepatoma cell line HepG2 obtained from the Riken Cell Bank (Ibaraki, Japan) were grown in Dulbecco's modified Eagle's medium 
(DMEM, Nissui Pharmaceutical Co., Ltd., Tokyo, Japan) supplemented with 10\% (v/v) fetal bovine serum (FBS). Human umbilical vein endothelial (Huve) cells obtained fromTaKaRa Bio Inc. (Shiga, Japan) were grown in MCDB107 medium (Cell Science \& Technology Institute, Inc., Miyagi, Japan) containing 10\% FBS, $10 \mathrm{ng} / \mathrm{mL}$ human epidermal growth factor (Sigma-Aldrich, Inc.), and $10 \mathrm{ng} / \mathrm{mL}$ human recombinant fibroblast growth factor-2 (ReproCELL, Kanagawa, Japan).

\subsection{Protein-BAM preparation}

Conjugate of four proteins, GFP, BSA, casein or ovalbumin, and BAM (protein-BAM) was prepared based on a previously reported method [4]. Briefly, phosphate-buffered saline (PBS, pH 7.4) containing $0.3 \%(\mathrm{w} / \mathrm{v})$ protein, and dimethyl sulfoxide containing $1.10 \%(\mathrm{w} / \mathrm{v})$ BAM-NHS were mixed at a molar ratio of protein to BAM-NHS of $1 / 10$. The mixture was then stirred for $2 \mathrm{~h}$ at room temperature and the resultant BAM-protein was purified through ultrafiltration for removing remaining free BAM-NHS. The product protein-BAM was dissolved in PBS and stored at $4^{\circ} \mathrm{C}$ until use.

\subsection{GFP-BAM anchoring}

The time-course profile of protein-BAM attachment to cells was investigated by suspending 10T1/2 cells at $4.0 \times 10^{5}$ cells $/ \mathrm{mL}$ in $200 \mu \mathrm{L}$ PBS containing $0.015,0.15$, or $1.5 \mu \mathrm{M}$ GFP-BAM. Protein-BAM concentrations refer to the above protein contents in this study. The cell fluorescence intensity, attributed to GFP attached to the cell surfaces, was measured using flow cytometry equipped with a laser and a filter allowing excitation and emission at 488 and $530 \mathrm{~nm}$, respectively (BD Accuri C6, BD Biosciences Co., San Jose, CA, USA). 


\subsection{ROS production}

Trypsinized 10T1/2 cells were soaked in DMEM containing $20 \mu \mathrm{M} \mathrm{BES}-\mathrm{H}_{2} \mathrm{O}_{2}-\mathrm{Ac}$ at $37^{\circ} \mathrm{C}$ in the dark for $1 \mathrm{~h}$, thus allowing reagent permeation into cells. After several rinses with PBS, cells were suspended for $15 \min$ in $200 \mu \mathrm{L}$ of PBS containing zero, $0.015,0.15$, or $1.5 \mu \mathrm{M}$ protein (BSA, casein, or ovalbumin)-BAM or containing BSA-bound oleic acid in $1.5 \mathrm{~mL}$ plastic tubes at $4.0 \times 10^{6}$ cells/tube. The oleic acid/BSA complex was prepared based on a report describing oleic acid effects on cellular ROS production [23]. Briefly, $26 \mathrm{~mL}$ of $4.5 \mathrm{mM}$ oleic acid in $150 \mathrm{mM}$ aqueous $\mathrm{NaCl}$ was dropped into $33 \mathrm{~mL}$ of $0.33 \mathrm{mM}$ BSA in $150 \mathrm{mM}$ aqueous $\mathrm{NaCl}$ to yield an oleic acid/BSA complex solution containing oleic acid and BSA at $2 \mathrm{mM}$ and $0.18 \mathrm{mM}$, respectively. Cells were then soaked in PBS containing $15 \mu \mathrm{M}$ oleic acid/BSA complex. The complex concentration was set based on the preparation conditions of BSA-BAM (molar ratio, 1/10) and the maximum BSA-BAM concentration at $1.5 \mu \mathrm{M}$ for cell treatments. Fluorescence attributed to the reaction of fluorescent ROS probe permeated into cells was determined using flow cytometry. Relative mean fluorescence intensities (RMFI), normalized using values obtained in cells soaked in PBS, were used for evaluating effects on ROS production resulting from anchoring protein-BAM to cell membranes. The dependence of effects on protein-BAM concentration and protein type was investigated using 10T1/2 cells. The dependency of these effects on cell type was investigated using HepG2 cells and Huve cells.

\subsection{ROS release}

For determining $\mathrm{H}_{2} \mathrm{O}_{2}$ release to the surrounding environment, $10 \mathrm{~T} 1 / 2$ cells were suspended in 200 $\mu \mathrm{L}$ of PBS containing zero or $1.5 \mu \mathrm{M}$ BSA-BAM in $1.5 \mathrm{~mL}$ tubes at $4.0 \times 10^{5}$ cells/tube for $10 \mathrm{~min}$. Then, the cells were suspended in PBS containing 1.0\% (w/v) Flu-Alg-Ph and/or $1.5 \mu \mathrm{M}$ HRP for 15 
min. In this step, $\mathrm{H}_{2} \mathrm{O}_{2}$ released from cells was consumed for the formation of a fluorescent sheath on an individual cell surface [26]. Subsequently, cells were rinsed several times with PBS and the fluorescent content of cells showing fluorescence, which was attributed to the existence of a Flu-Alg-Ph sheath, was determined by flow cytometry.

\subsection{Cytotoxicity assessment}

Cytotoxicity induced by BAM anchoding was evaluated by trypan blue exclusion after the treaemtnt, subsequent growth, and clonogenic acssay for 10T1/2, HepG2, and Huve cells. The cells were soaked in PBS containing 1.5 $\mu \mathrm{M}$ BSA-BAM for $15 \mathrm{~min}$. Then, cell viabilities were measured by trypan blue exclusion using a hemocytometer. Adverse effects that required time to induce cell death and growth inhibition were determined by seeding cells at $5.0 \times 10^{4}$ (viable cells)/well in $60-\mathrm{mm}$ diameter tissue culture dishes. Cell growth profiles were then measured after 3 days using trypsinization and a hemocytometer. Doubling time was calculated from cell number increases of cells from day 1 to 3 . Cells soaked in PBS were used as controls and designated as "untreated cells." Clonogenic assay that measures the ability of single cells to form colonies was performed based on the reported method by Fede et al [29]. The treated cells were seeded at 300 cells/well in 60-mm diameter tissue culture dishes. After 3-5 days of culture, the colonies contianing more than 50 cells were counted after staining with cystal violet. Cloning efficiency was calculated by normalizing the data of treated cells against the average values of untreated cells.

\subsection{Statistical analysis}


Quantitative data were expressed as the mean \pm standard deviation (SD) of triplicate or quadruplicate experiments. Comparisons among two data sets and more than two data sets were made using the Student's paired $t$-test and one-way ANOVA with Tukey's post hoc analysis, respectively. Values of $p<0.05$ were considered to indicate significance.

\section{Results}

\subsection{GFP-BAM anchoring}

GFP-BAM was employed for evaluating the time-course profile of protein-BAM attachment to cell surfaces. No obvious increase in fluorescence intensity, attributed to the existence of GFP, was observed in cells soaked in $0.015 \mu \mathrm{M}$ GFP-BAM solution compared with those before treatment (Fig. 2). GFP-BAM concentration that was dependent on increases in fluorescence was detected in cells soaked in 0.15 and $1.5 \mu \mathrm{M}$ GFP-BAM solutions. The observed fluorescence showed constant values at 1 and 10 min of soaking in 0.15 and $1.5 \mu \mathrm{M}$ GFP-BAM solutions, respectively. The fluorescence attributed to GFP was observed in the solution after 15 min of soaking cells in $1.5 \mu \mathrm{M}$ GFP-BAM

solution. Based on the result, we decided the maximum concentration of protein-BAM $(1.5 \mu \mathrm{M})$ and soaking time (15 $\mathrm{min})$ in the following experiments.

\subsection{Dependency on protein-BAM concentration}

BSA-BAM and 10T1/2 cells were used for evaluating the dependency of ROS production on protein-BAM concentration. ROS production was evaluated after soaking cells in solutions containing 
protein-BAM for 15 min (Fig. 2). Notable increases in ROS production were not observed in cells at 1 min of soaking in $0.015,0.15$, and $1.5 \mu \mathrm{M}$ BSA-BAM solutions, compared with those of untreated cells (Fig. 3). After 15 min of soaking, RMFI, arising from intracellular fluorescent dye converted from fluorescent probe of cells soaked in $0.015 \mu \mathrm{M}$ BSA-BAM solution, did not increase compared with that at 1 min of soaking. In contrast, RMFI of cells soaked in 0.15 and $1.5 \mu \mathrm{M}$ BSA-BAM solutions increased 16\% and 76\%, respectively (both $p<0.01$ ). The effects of soaking cells in solutions containing BSA or oleic acid/BSA complex were also evaluated and no significant increases in RMFIs were observed in cells soaked in $1.5 \mu \mathrm{M}$ BSA solution. In contrast, a significant increase was observed in those soaked in oleic acid/BSA complex solution containing $15 \mu \mathrm{M}$ oleic acid and $1.4 \mu \mathrm{M}$ BSA $(p=0.03)$.

\subsection{Effect of conjugated protein with BAM}

The independence of ROS production enhancement on conjugated protein with BAM was evaluated using casein and ovalbumin. The obtained trends were the same with those obtained in cells soaked in BSA and BSA-BAM solutions. No significant increases in RMFIs were detected in cells soaked in 1.5 $\mu \mathrm{M}$ casein and ovalbumin solutions (Fig. 4). In contrast, RMFIs increased after 15 min of soaking in $1.5 \mu \mathrm{M}$ casein-BAM $(p<0.01)$ and ovalbumin-BAM solutions $(p=0.01)$.

\subsection{Effects of cell type}

The independence of ROS production stimulation on cell type was evaluated using HepG2 and Huve cells. The degrees of ROS production enhancement were not the same, but both cell types increased in 
ROS production with BSA-BAM treatment (Fig. 5). RMFIs after 15 min of soaking in BSA-BAM solution were 13 and 27\% (both $p<0.01$ ) higher in HepG2 and Huve cells, respectively, than those of untreated cells.

\section{5. $\mathrm{H}_{2} \mathrm{O}_{2}$ release}

The effects of protein-BAM attachment on ROS released to the surrounding environment were also examined. $\mathrm{H}_{2} \mathrm{O}_{2}$ release was evaluated using flow cytometry to measure the fluorescence of cells covered with Flu-Alg-Ph sheaths formed through HRP-catalyzed cross-linking of Flu-Alg-Ph molecules that consumed $\mathrm{H}_{2} \mathrm{O}_{2}$ released from individual cells [26]. Sequential soaking of cells in BSA-BAM and then Flu-Alg-Ph solutions did not induce cell fluorescence higher than the threshold value obtained for untreated cells (Fig. 6a). Soaking cells in $\mathrm{HRP}+$ Flu-Alg-Ph solution without soaking in BSA-BAM solution resulted in $8 \%$ of cells showing fluorescence attributed to Flu-Alg-Ph. These values increased to $17 \%$ in cells soaked sequentially in BSA-BAM solution and HRP + Flu-Alg-Ph solution.

\subsection{Cytotoxicity assessment}

The cytotoxic effect of anchoring protein-BAM on cells was evaluated by measuring cell viability, growth, and colony formation of 10T1/2, HepG2, and Huve cells. No obvious adverse effects resulting from BAM-BSA attachment were observed for all these cells. The viabilities of cells soaked in $1.5 \mu \mathrm{M}$ BSA-BAM were $>90 \%$ (Table 1). No notable differences in morphology were found in cells attached to cell culture dishes compared with untreated cells (Fig. 7) and they grew with doubling times similar 
to those of untreated cells (Table 1). No decrease in the ability of colony formation by single cells compared with untreated cells was also found for all the cells (Table 1).

\section{Discussion}

The purpose of this study was to investigate the effect of BAM-anchoring to cell membranes on ROS production. Considering BAM effectiveness in a variety of cell studies, the knowledge obtained in this study would be useful for understanding cell behavior results in such applications because of the association of ROS with a variety of cellular events including those induced by DNA damage [11-17, 30]. First, the attachment profile of protein-BAM to cell surfaces was evaluated using GFP-BAM. GFP was chosen here for its ease of evaluation by fluorescence. The results demonstrated that protein-BAM attachment was rapid (Fig. 2), which was in agreement with reports dealing with BAM conjugated with fluorescein, a small molecule [4], and demonstrated the independence of rapid anchoring on the conjugated substrate sizes with BAM.

Next, the possibility of ROS production enhancement was evaluated using 10T1/2 cells. The similar degree of ROS production stimulation by $1.5 \mu \mathrm{M}$ BSA-BAM, casein-BAM and ovalbumin-BAM, and no effect by the proteins themselves (Figs. 3, 4), clearly indicated stimulation of ROS production by BAM-anchoring to cells. Regarding the effect of protein-BAM concentration, no significant ROS production increase in cells treated with $0.015 \mu \mathrm{M}$ BSA-BAM (Fig. 3) was considered here to result from homeostatic adjustments toward the applied stimulation. It is known that intracellular ROS generation and scavenging are regulated by several biological mechanisms [31, 32]. BSA-BAM dose-dependent increases in ROS production observed here with 0.15 and $1.5 \mu \mathrm{M}$ BSA-BAM treatments would have been because the homeostatic system was overwhelmed. Enhancement of ROS 
production observed not only in 10T1/2 cells but also HepG2 and Huve cells (Fig. 5) supported the hypothesis that ROS production was stimulated by BAM anchored to cell membranes. The differences in the degree of ROS production enhancement between 10T1/2, HepG2, and Huve cells ( $93 \%, 13 \%$, and $27 \%$, respectively) compared with those of untreated cells, were attributed to cellular diversity. Servettaz et al. have reported ROS production response differences to stimulation by sera from unhealthy patients for Huve, mouse fibroblast, and human cancer cells [33]. Chang et al. have reported differences in response to ROS stimulation by polymeric materials for human gingival epithelial cells and pulp fibroblasts [34]. The lowest degree of ROS production stimulation in HepG2 cells would have been because of a highly expressed ROS scavenging system in hepatic cells [35]. A possible mechanism of the stimulation of ROS production by BAM-anchoring to cell membrane is as follows. The oleyl groups anchored to cell membrane induced rearrangement of the membrane lipid structure and in turn, enhanced ROS production through the increase in enzyme activity of NADPH in cell membranes. It is known that the incorporation of oleic acid into cell membrane can rearrange the membrane lipid structure, including lipid rafts through the interaction of oleic acid with membrane lipids [36]. Wang et al reported that lipolysis products of triglyceride-rich lipoprotein induced aggregation of endothelial cell membrane lipid rafts and enhanced ROS production through an activation of NADPH oxidase in cell membrane [37]. NADPH oxidases are membrane enzyme complexes that produce ROS [38]. The enhancement of ROS production is not specific to the rearrangement of membrane components through the direct interaction with membrane lipids, similar results were reported on the rearrangement of membrane poroteins by their cross-linking through the formation of avidin-biotin complex for erythrocytes and neutrophils $[39,40]$.

The results of ROS release study (Fig. 6) demonstrated that BAM-anchoring increased ROS release to the surrounding environment while increasing ROS production. The increase in ROS release is not 
specific for BAM-anchoring, as similar results have been reported for cells treated with oleic acid [22, 24]. A concern for these results might have been the $7.6 \%$ of cells showing fluorescence despite the absence of BSA-BAM. This result could be explained by the existence of a cell fraction that constitutively released ROS in sufficient amounts for formation of Flu-Alg-Ph sheaths through the HRP-catalyzed reaction; ROS are formed as a natural byproduct of aerobic metabolism [41]. By combining the results of intracellular ROS production (Figs. 3-5) and ROS release to the surrounding environment (Fig. 6), it was demonstrated here the necessity for focusing attention on cell behavior. Cells free from BAM-anchoring, yet existing in the same system with cells treated with BAM, need to be considered as well as those treated with BAM. Addition of ROS scavenging chemicals in media, such as catalase, is a possible means for suppressing the unintended effect of cellular-released ROS.

Important results developed here, which support the reported superior biocompatibility of BAM [4-7], were that all cells maintained superior viability (>90\%) after BAM-anchoring under conditions that induced significantly increased ROS production. In addition, these cells grew and had the ability of colony formation in a manner similar to untreated cells after seeding on cell culture dishes (Table 1) despite the fact that oxidative stress has the potential to induce cell cycle arrest, resulting in suppression of cellular growth [42]. The degree of ROS production stimulation observed here, induced by BAM-anchoring, was insufficient to induce cell death and growth inhibition. These results indicated unremarkable effects from BAM-anchoring on cell viability and growth, which was acceptable considering that oleic acid is the most widely distributed fatty acid and abundant in nature, being found in animal fats and vegetable oils. In future studies of using certain cells for specific purposes, we will explore the effects on cellular functions including the damage to DNA which will govern the success. Apart from the stimulation of ROS production, the potency of induction of immunological reactions by PEG moiety [43] should be paid attention in the applications of BAM (PEG-oleate) in vivo. 


\section{Conclusion}

The functions of ROS on regulation of a variety of cellular events are well known. In this study, stimulation of intracellular ROS production by BAM was investigated because BAM contains an oleyl group and oleic acid has been reported as an inducer of ROS production [22-25]. Enhancement of intracellular ROS generation was confirmed here with 0.15 and $1.5 \mu \mathrm{M}$ protein-BAM treatments. Enhancement of $\mathrm{H}_{2} \mathrm{O}_{2}$ release from treated cells to the surrounding environment was also confirmed. Despite stimulated ROS generation, no adverse effects on viability and growth of treated cells were observed. These results support grounds for the biocompatibility of BAM treatment, based on the viability and growth of treated cells [4-7]. However, results obtained for ROS generation and release demonstrated the necessity for focusing attention on BAM effects for correctly understanding results in the studies applying BAM to cells.

\section{Acknowledgments}

This research was supported by the Japan Society for the Promotion of Science KAKENHI grant (No. 26630428) from the Ministry of Education, Culture, Sports, Science and Technology of Japan (MEXT) and the Adaptable and Seamless Technology Transfer Program through Target-driven R\&D (No. AS262Z00383P), Japan Science and Technology Agency.

\section{References}

[1] Y. Kurishita, T. Kohira, A. Ojida, I. Hamachi, Organelle-localizable fluorescent chemosensors for site-specific 
multicolor imaging of nucleoside polyphosphate dynamics in living cells, J. Am. Chem. Soc., 134 (2012) 18779-18789.

[2] S.T. Selvan, P.K. Patra, C.Y. Ang, J.Y. Ying, Synthesis of silica-coated semiconductor and magnetic quantum dots and their use in the imaging of live cells, Angew. Chem. Int. Ed. Engl., 46 (2007) 2448-2452.

[3] H.A. Chung, K. Kato, C. Itoh, S. Ohhashi, T. Nagamune, Casual cell surface remodeling using biocompatible lipid-poly(ethylene glycol)(n): development of stealth cells and monitoring of cell membrane behavior in serum-supplemented conditions, J. Biomed. Mater. Res. A, 70 (2004) 179-185.

[4] K. Kato, C. Itoh, T. Yasukouchi, T. Nagamune, Rapid protein anchoring into the membranes of mammalian cells using oleyl chain and poly(ethylene glycol) derivatives, Biotechnol. Prog., 20 (2004) 897-904.

[5] S. Sakai, M. Taya, On-cell surface cross-linking of polymer molecules by horseradish peroxidase anchored to cell membrane for individual cell encapsulation in hydrogel sheath, ACS Macro Lett, 3 (2014) 972-975.

[6] K. Kato, K. Umezawa, D.P. Funeriu, M. Miyake, J. Miyake, T. Nagamune, Immobilized culture of nonadherent cells on an oleyl poly(ethylene glycol) ether-modified surface, Biotechniques, 35 (2003) 1014-1018, $1020-1011$.

[7] Y. Shimizu, T. Kihara, S.M. Haghparast, S. Yuba, J. Miyake, Simple display system of mechanical properties of cells and their dispersion, PLoS One, 7 (2012) e34305.

[8] R. Kawamura, M. Mishima, S. Ryu, Y. Arai, M. Okose, Y.R. Silberberg, S.R. Rao, C. Nakamura, Controlled cell adhesion using a biocompatible anchor for membrane-conjugated bovine serum albumin/bovine serum albumin mixed layer, Langmuir, 29 (2013) 6429-6433.

[9] H. Yamazoe, T. Ichikawa, Y. Hagihara, Y. Iwasaki, Generation of a patterned co-culture system composed of adherent cells and immobilized nonadherent cells, Acta Biomater., 31 (2016) 231-240.

[10] S.T. Selvan, P.K. Patra, C.Y. Ang, J.Y. Ying, Synthesis of silica-coated semiconductor and magnetic quantum dots and their use in the imaging of live cells, Angew. Chem. Int. Ed. Engl., 46 (2007) 2448-2452. 
[11] D. Nikitovic, E. Corsini, D. Kouretas, A. Tsatsakis, G. Tzanakakis, ROS-major mediators of extracellular matrix remodeling during tumor progression, Food Chem. Toxicol., 61 (2013) 178-186.

[12] T.R. Hurd, M. DeGennaro, R. Lehmann, Redox regulation of cell migration and adhesion, Trends Cell Biol., $22(2012) 107-115$.

[13] W.H. Liu, L.S. Chang, Caffeine induces matrix metalloproteinase-2 (MMP-2) and MMP-9 down-regulation in human leukemia U937 cells via $\mathrm{Ca}^{2+} / \mathrm{ROS}-$ mediated suppression of ERK/c-fos pathway and activation of p38 MAPK/c-jun pathway, J. Cell. Physiol., 224 (2010) 775-785.

[14] J. Liu, Q.W. Ben, W.Y. Yao, J.J. Zhang, D.F. Chen, X.Y. He, L. Li, Y.Z. Yuan, BMP2 induces PANC-1 cell invasion by MMP-2 overexpression through ROS and ERK, Front Biosci (Landmark Ed), 17 (2012) 2541-2549.

[15] A. Gupta, S.F. Rosenberger, G.T. Bowden, Increased ROS levels contribute to elevated transcription factor and MAP kinase activities in malignantly progressed mouse keratinocyte cell lines, Carcinogenesis, 20 (1999) 2063-2073.

[16] H.Y. Li, S.Y. Wu, N. Shi, Transcription factor Nrf2 activation by deltamethrin in PC12 cells: Involvement of ROS, Toxicol. Lett., 171 (2007) 87-98.

[17] M.A. Verity, D.E. Bredesen, T. Sarafian, Role of reactive oxygen species (ROS) in neuronal degeneration. Modulation by protooncogene expression, Ann. N. Y. Acad. Sci., 765 (1995) 340.

[18] D.P. Enot, M. Niso-Santano, S. Durand, A. Chery, F. Pietrocola, E. Vacchelli, F. Madeo, L. Galluzzi, G. Kroemer, Metabolomic analyses reveal that anti-aging metabolites are depleted by palmitate but increased by oleate in vivo, Cell Cycle, 14 (2015) 2399-2407.

[19] A. Linos, E. Kaklamanis, A. Kontomerkos, Y. Koumantaki, S. Gazi, G. Vaiopoulos, G.C. Tsokos, P. Kaklamanis, The effect of olive oil and fish consumption on rheumatoid arthritis--a case control study, Scand. J. Rheumatol., 20 (1991) 419-426.

[20] H.G. Rodrigues, M.A. Vinolo, J. Magdalon, K. Vitzel, R.T. Nachbar, A.F. Pessoa, M.F. dos Santos, E. 
Hatanaka, P.C. Calder, R. Curi, Oral administration of oleic or linoleic acid accelerates the inflammatory phase of wound healing, J. Invest. Dermatol., 132 (2012) 208-215.

[21] L. Lipworth, M.E. Martinez, J. Angell, C.C. Hsieh, D. Trichopoulos, Olive oil and human cancer: an assessment of the evidence, Prev. Med., 26 (1997) 181-190.

[22] E. Hatanaka, A. Dermargos, A.E. Hirata, M.A. Vinolo, A.R. Carpinelli, P. Newsholme, H.A. Armelin, R. Curi, Oleic, linoleic and linolenic acids increase ros production by fibroblasts via NADPH oxidase activation, PLoS One, 8 (2013) e58626.

[23] H. Gremmels, L.M. Bevers, J.O. Fledderus, B. Braam, A.J. van Zonneveld, M.C. Verhaar, J.A. Joles, Oleic acid increases mitochondrial reactive oxygen species production and decreases endothelial nitric oxide synthase activity in cultured endothelial cells, Eur. J. Pharmacol., 751 (2015) 67-72.

[24] E. Hatanaka, A.C. Levada-Pires, T.C. Pithon-Curi, R. Curi, Systematic study on ROS production induced by oleic, linoleic, and gamma-linolenic acids in human and rat neutrophils, Free Radic. Biol. Med., 41 (2006) $1124-1132$.

[25] G. Lu, E.L. Greene, T. Nagai, B.M. Egan, Reactive oxygen species are critical in the oleic acid-mediated mitogenic signaling pathway in vascular smooth muscle cells, Hypertension, 32 (1998) 1003-1010.

[26] Y. Liu, S. Sakai, S. Kawa, M. Taya, Identification of hydrogen peroxide-secreting cells by cytocompatible coating with a hydrogel membrane, Anal. Chem., 86 (2014) 11592-11598.

[27] T. Ashida, Y. Ojima, S. Sakai, M. Sakka, K. Sakka, K. Kawakami, M. Taya, Designing fusion proteins with carbohydrate-binding modules having affinity to enzymatically gellable carboxymethylcellulose derivative hydrogel, J. Chem. Eng. Jpn., 47 (2014) 835-840.

[28] S. Sakai, K. Kawakami, Synthesis and characterization of both ionically and enzymatically crosslinkable alginate, Acta Biomater., 3 (2007) 495-501.

[29] C. Fede, F. Selvestrel, C. Compagnin, M. Mognato, F. Mancin, E. Reddi, L. Celotti, The toxicity outcome of 
silica nanoparticles $(\operatorname{Ludox}(\mathrm{R}))$ is influenced by testing techniques and treatment modalities, Anal. Bioanal. Chem., 404 (2012) 1789-1802.

[30] N.R. Jena, DNA damage by reactive species: Mechanisms, mutation and repair, J. Biosci., 37 (2012) $503-517$.

[31] R.C. Zangar, D.R. Davydov, S. Verma, Mechanisms that regulate production of reactive oxygen species by cytochrome P450, Toxicol. Appl. Pharmacol., 199 (2004) 316-331.

[32] P. Jezek, L. Hlavata, Mitochondria in homeostasis of reactive oxygen species in cell, tissues, and organism, Int. J. Biochem. Cell Biol., 37 (2005) 2478-2503.

[33] A. Servettaz, P. Guilpain, C. Goulvestre, C. Chereau, C. Hercend, C. Nicco, L. Guillevin, B. Weill, L. Mouthon, F. Batteux, Radical oxygen species production induced by advanced oxidation protein products predicts clinical evolution and response to treatment in systemic sclerosis, Ann. Rheum. Dis., 66 (2007) 1202-1209.

[34] H.H. Chang, M.K. Guo, F.H. Kasten, M.C. Chang, G.F. Huang, Y.L. Wang, R.S. Wang, J.H. Jeng, Stimulation of glutathione depletion, ROS production and cell cycle arrest of dental pulp cells and gingival epithelial cells by HEMA, Biomaterials, 26 (2005) 745-753.

[35] M.E. Kyle, D. Nakae, I. Sakaida, S. Miccadei, J.L. Farber, Endocytosis of superoxide dismutase is required in order for the enzyme to protect hepatocytes from the cytotoxicity of hydrogen peroxide, J. Biol. Chem., 263 (1988) 3784-3789.

[36] S. Lopez, B. Bermudez, S. Montserrat-de la Paz, S. Jaramillo, L.M. Varela, A. Ortega-Gomez, R. Abia, F.J. Muriana, Membrane composition and dynamics: a target of bioactive virgin olive oil constituents, Biochim. Biophys. Acta, 1838 (2014) 1638-1656.

[37] L. Wang, A.R. Sapuri-Butti, H.H. Aung, A.N. Parikh, J.C. Rutledge, Triglyceride-rich lipoprotein lipolysis increases aggregation of endothelial cell membrane microdomains and produces reactive oxygen species, Am. J. Physiol. Heart Circ. Physiol., 295 (2008) H237-244. 
[38] J. Han, V.V. Shuvaev, V.R. Muzykantov, Targeted interception of signaling reactive oxygen species in the vascular endothelium, Ther. Deliv., 3 (2012) 263-276.

[39] V.R. Muzykantov, M.D. Smirnov, G.P. Samokhin, Avidin-induced lysis of biotinylated erythrocytes by homologous complement via the alternative pathway depends on avidins ability of multipoint binding with biotinylated membrane, Biochim. Biophys. Acta, 1107 (1992) 119-125.

[40] V.R. Muzykantov, T.A. Kushnareva, M.D. Smirnov, E.K. Ruuge, Avidin attachment to biotinylated human neutrophils induces generation of superoxide anion, Biochim. Biophys. Acta, 1177 (1993) 229-235.

[41] M. Giorgio, M. Trinei, E. Migliaccio, P.G. Pelicci, Hydrogen peroxide: a metabolic by-product or a common mediator of ageing signals?, Nat. Rev. Mol. Cell Biol., 8 (2007) 722-728.

[42] R.E. Shackelford, W.K. Kaufmann, R.S. Paules, Oxidative stress and cell cycle checkpoint function, Free Radic. Biol. Med., 28 (2000) 1387-1404.

[43] H. Schellekens, W.E. Hennink, V. Brinks, The immunogenicity of polyethylene glycol: facts and fiction, Pharm. Res., 30 (2013) 1729-1734. 


\section{Figure Captions}

Fig. 1. Schematic illustration of BAM-anchoring to a cell membrane.

Fig. 2. Fluorescence intensity increase ratios in cells arising from GFP conjugated with BAM, measured by flow cytometry. Cells were soaked in $0.015(\mathbf{\bullet}), 0.15(\bullet)$, or $1.5 \mu \mathrm{M}(\mathbf{\Delta})$ BSA-BAM solution. Bars, $\mathrm{SD}(n=3)$.

Fig. 3. Transition of relative mean fluorescence intensities (RMFIs) arising from intracellular fluorescent dye converted from fluorescent probe with time measured using a flow cytometer. 10T1/2c cells were soaked in solutions containing (a) BSA-BAM (white column, $0.015 \mu \mathrm{M}$; grey column, 0.15 $\mu \mathrm{M}$; and black column, $1.5 \mu \mathrm{M})$, (b) BSA $(1.5 \mu \mathrm{M})$, or (c) oleic acid/BSA (complex of $15 \mu \mathrm{M}$ oleic acid and $1.4 \mu \mathrm{M}$ BSA) solution. RMFIs were normalized using the values obtained from cells soaked in PBS at the corresponding time points. Bars, $\mathrm{SD}(n=4) ;+, p<0.05$ vs cells soaked in PBS; and \#, $p<0.05$ vs cells soaked in the same solution for $1 \mathrm{~min}$.

Fig. 4. Transition of RMFIs attributed to intracellular fluorescent dye converted from fluorescent probe with time measured using a flow cytometry. 10T1/2 cells were soaked in solutions containing (a) 1.5 $\mu \mathrm{M}$ casein (white column) or $1.5 \mu \mathrm{M}$ casein-BAM (black column), or (b) $1.5 \mu \mathrm{M}$ ovalbumin (white column) or $1.5 \mu \mathrm{M}$ ovalbumin-BAM (black column). RMFIs were normalized using the values obtained from cells soaked in PBS at the corresponding time points. Bars, $\mathrm{SD}(n=4) ;+, p<0.05$ vs cells soaked in PBS; and \#, $p<0.05$ vs cells soaked in the corresponding solution for $1 \mathrm{~min}$. 
Fig. 5. Transition of RMFIs arising from intracellular fluorescent dye converted from fluorescent probe with time measured by flow cytometry. (a) HepG2 and (b) Huve cells were soaked in solutions containing $1.5 \mu \mathrm{M}$ BSA (white column) or $1.5 \mu \mathrm{M}$ BSA-BAM (black column). RMFIs were normalized using the values obtained from cells soaked in PBS at the corresponding time points. Bars, SD $(n=4) ;+, p<0.05$ vs cells soaked in PBS; and \#, $p<0.05$ vs cells soaked in the same solution for 1 $\min$.

Fig. 6. Effects of BSA-BAM treatment on cell population showing higher fluorescence intensity, attributed to the existence of Flu-Alg-Ph sheaths, than the threshold value determined from untreated cell data. Bars, $\operatorname{SD}(n=3)$.

Fig. 7. Microphotographs of $10 \mathrm{~T} 1 / 2, \mathrm{HepG} 2$, and Huve cells, untreated and treated with $1.5 \mu \mathrm{M}$ BSA-BAM, at 1 day after seeding on cell culture dishes. Magnification, $\times 100$ and bars, $100 \mu \mathrm{m}$. 
Table 1. Viability of cells treated with BSA-BAM and doubling time of untreated and treated cells.

\begin{tabular}{llll}
\hline & $10 \mathrm{~T} 1 / 2$ cells & HepG2 cells & Huve cells \\
\hline Viability (\%) & $95.7 \pm 1.7$ & $93.6 \pm 0.2$ & $91.3 \pm 0.4$ \\
$\begin{array}{c}\text { Doubling time (h) } \\
\text { Untreated }\end{array}$ & $14.2 \pm 0.5$ & $34.5 \pm 1.5$ & $31.5 \pm 3.9$ \\
BSA-BAM & $14.9 \pm 0.8(p=0.25)$ & $33.4 \pm 1.7(p=0.44)$ & $26.2 \pm 2.3(p=0.11)$ \\
Cloning efficiency & $104.7 \pm 10.3$ & $95.0 \pm 17.8$ & $100.5 \pm 9.4$ \\
$(\%$ untreared cells $)$ & $(p=0.42)$ & $(p=0.64)$ & $(p=0.96)$ \\
\hline
\end{tabular}

Data represent mean $\pm \mathrm{SD}$ ( $n=3$ for viability and doubling time, $n=6$ for cloning efficiency), Doubling times were deteremined in the cultures of respective cells during 1-3 days, and $p$-values mean comparison with untreated cells. 
Fig. 1

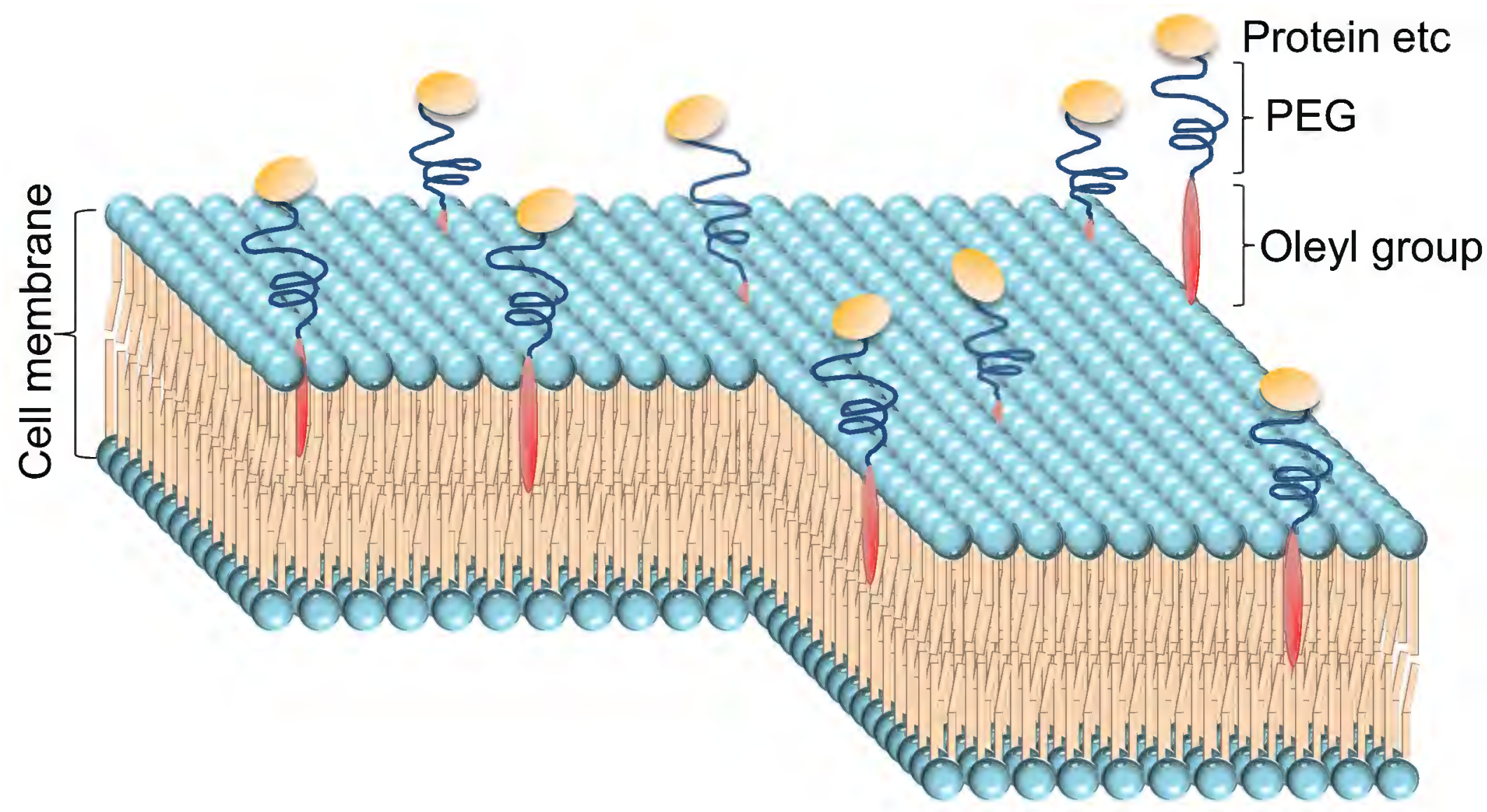


Fig. 2

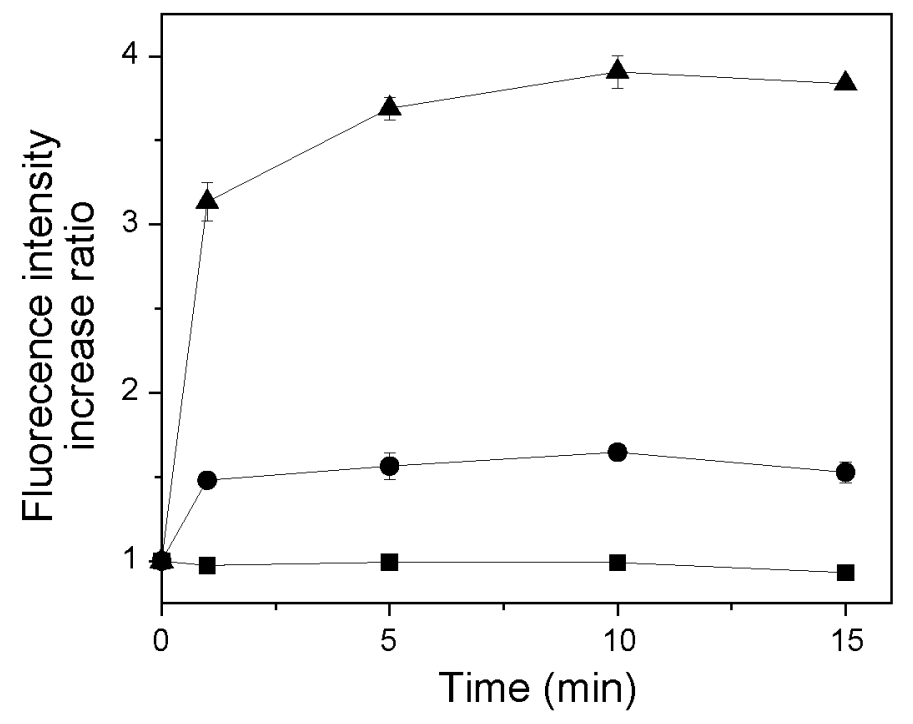


Fig. 3

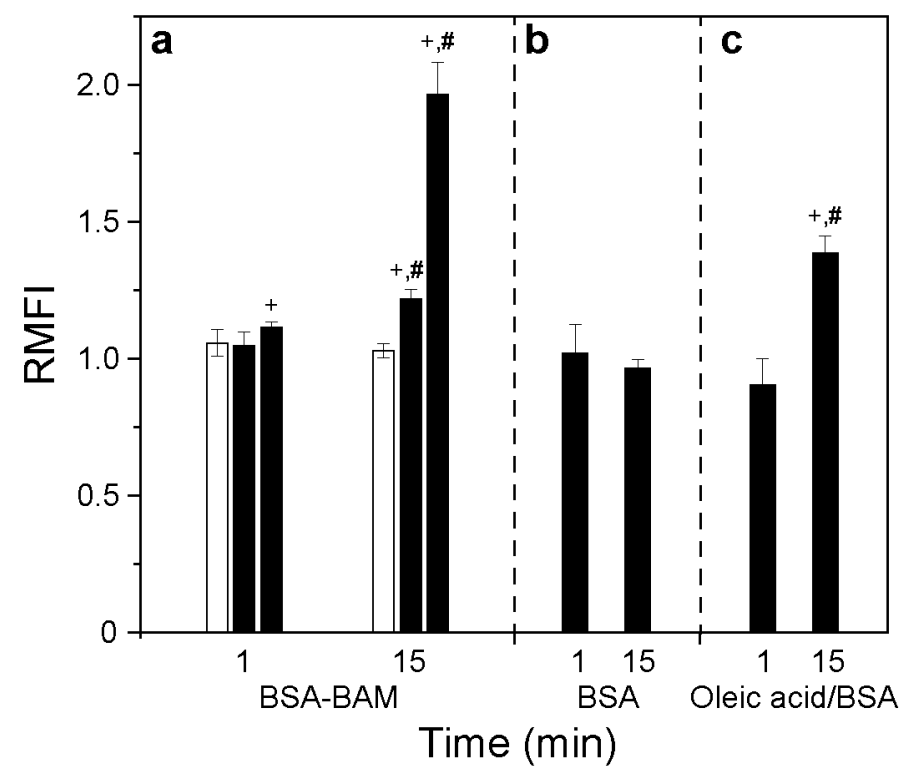


Fig. 4

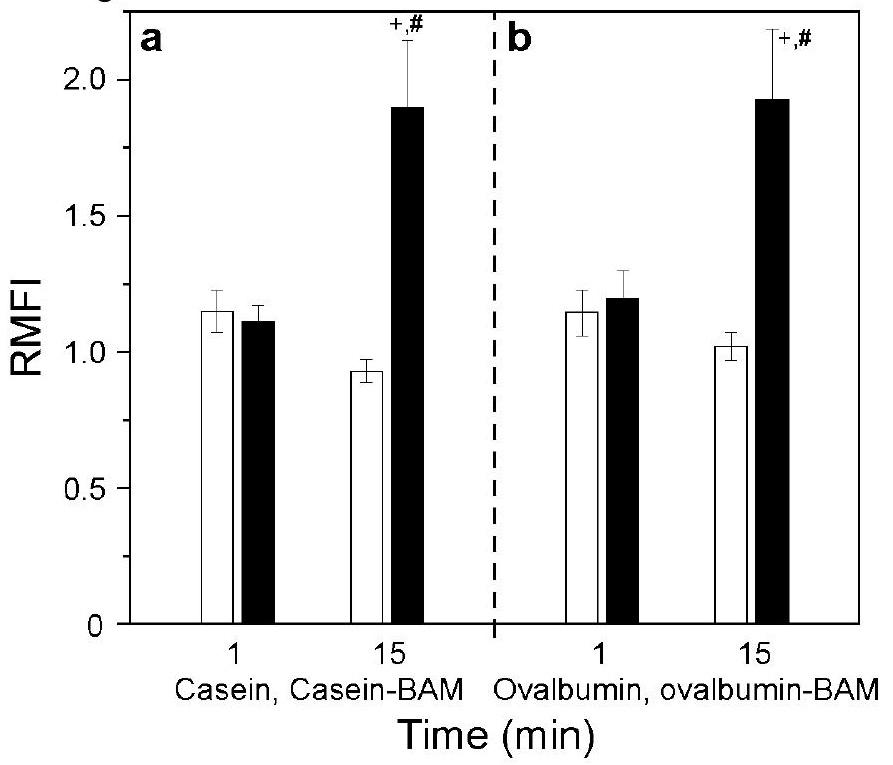


Fig. 5

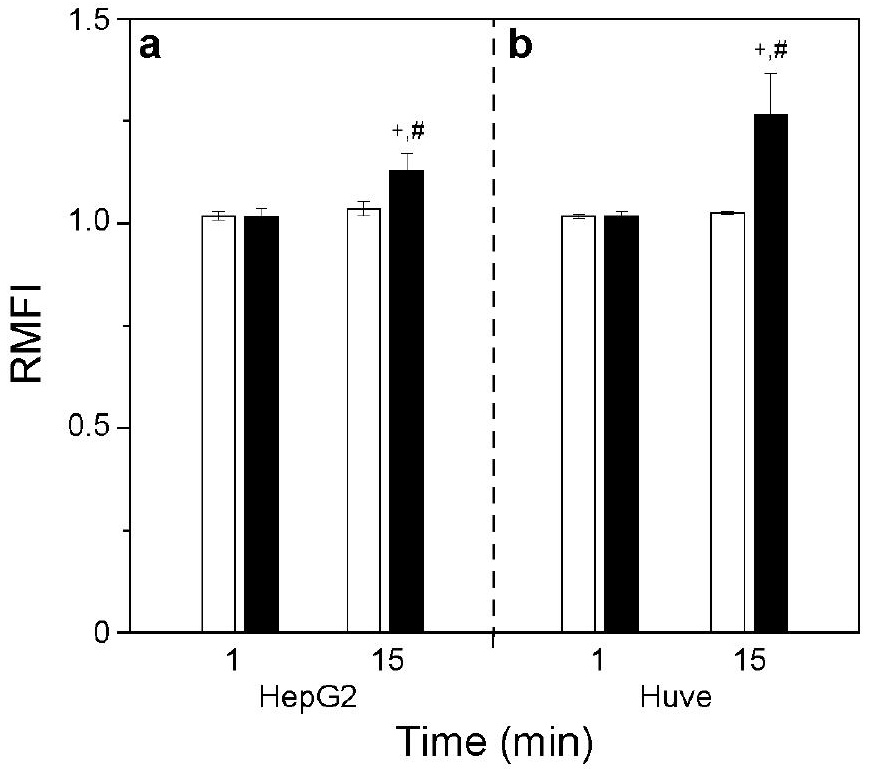


Fig. 7

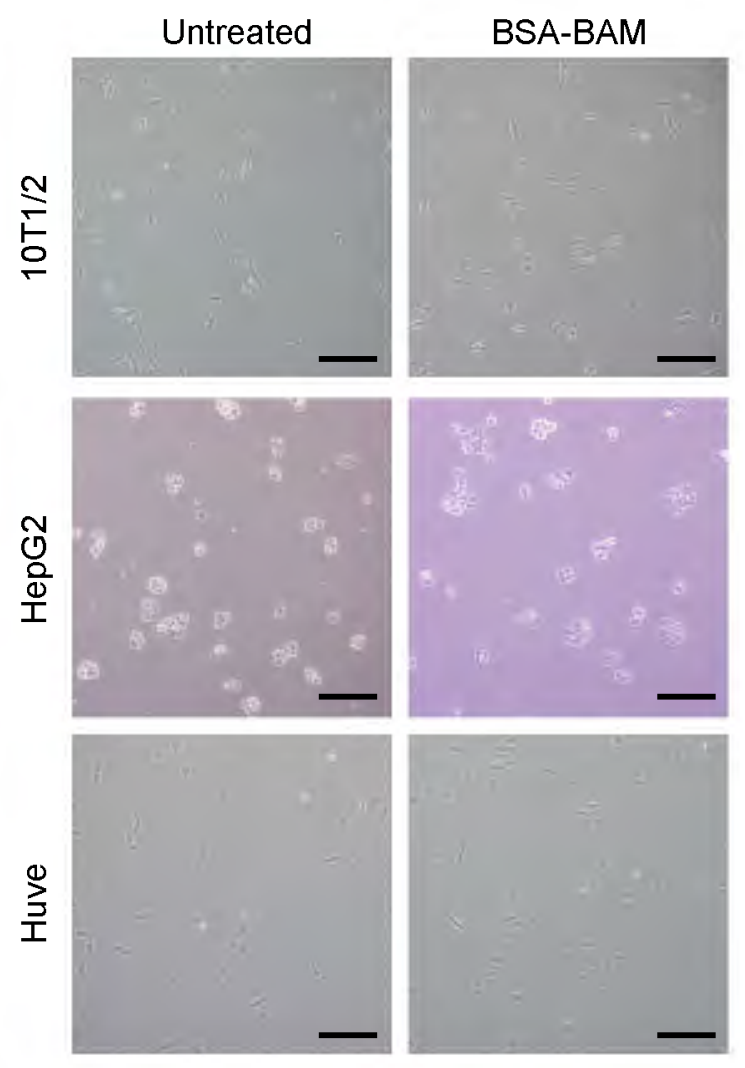




\section{Graphical abstract}

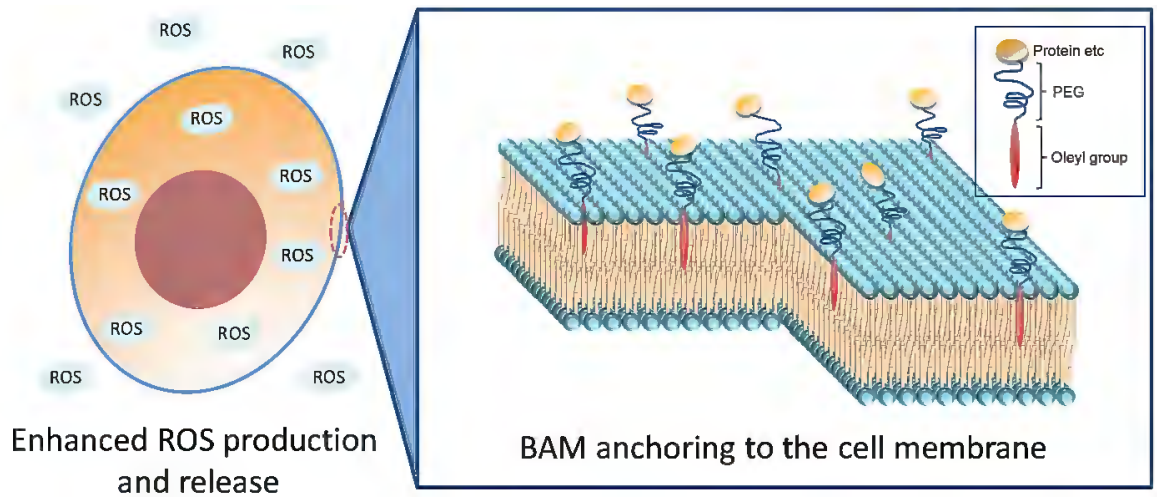

